\title{
Effects of de-escalated bisphosphonate therapy on bone turnover biomarkers in breast cancer patients with bone metastases
}

\author{
Christina L Addison ${ }^{1,2,3^{*}}$, Gregory R Pond ${ }^{4}$, Huijun Zhao ${ }^{1}$, Sasha Mazzarello ${ }^{1}$, Lisa Vandermeer ${ }^{1}$, Robyn Goldstein ${ }^{5}$,
} Eitan $\mathrm{Amir}^{5}$ and Mark Clemons ${ }^{1,2}$

\begin{abstract}
While de-escalation of bisphosphonates from 4 to 12-weekly dosing has been shown to be clinically non-inferior to standard dosing, there is evidence the de-escalation is associated with increased bone turnover biomarkers. Here we evaluated the effect of de-escalated dosing on a panel of biomarkers and determined their association with incidence of skeletal related events (SREs) in breast cancer patients with 'low risk' bone metastases. As part of a pilot randomized trial, women with baseline C-telopeptide levels $<600 \mathrm{ng} / \mathrm{L}$ after $>3$ months of 3-4 weekly pamidronate were randomized to continue pamidronate every 4 weeks or de-escalation to 12-weekly treatment. Serum was analysed for bone biomarkers (C-telopeptide, N-telopeptide, bone-specific alkaline phosphatase, transforming growth factor- $\beta$, procollagen type $1 \mathrm{~N}$-propeptide, activinA and bone sialoprotein) using ELISA. The associations between changes in biomarkers, pain scores and SREs were assessed by univariable logistic regression. Numerical increases in all biomarkers were observed between baseline and 12 weeks but were of higher magnitude in the de-escalated arm. Pain scores in the de-escalated treatment arm showed a greater magnitude of pain reduction from baseline to 12 weeks. Neither baseline levels nor changes in biomarkers from baseline to 12 weeks on treatment were associated with on study SREs. Baseline pain as measured by the FACT-BP was associated with increased risk of SRE. In conclusion, biomarkers of bone activity do not appear to predict for SREs in 'low risk' cohorts. However, baseline bone pain appears to be associated with SRE occurrence, a finding which warrants evaluation in larger cohorts.
\end{abstract}

Keywords: Bisphosphonate; Bone metastasis; Breast cancer; Biomarker; Skeletal related event; De-escalated therapy

\section{Introduction}

Bone is the most common site of metastasis in breast cancer (Coleman \& Rubens 1987) and skeletal metastases are associated with skeletal related events (SREs) such as; surgery/radiation to bone, pathological fractures, spinal cord compression and hypercalcemia. Development of SREs can significantly impair a patient's quality of life, and thus current treatment strategies attempt to prevent or delay the occurrence of SREs. As the osteoclast is the primary cell type implicated in bone destruction, osteoclast-inhibiting agents such as bisphosphonates or denosumab are essential components of the management

\footnotetext{
* Correspondence: caddison@ohri.ca

${ }^{1}$ Program for Cancer Therapeutics, Ottawa Hospital Research Institute, Ottawa, ON, Canada

2Department of Medicine, University of Ottawa, Ottawa, ON, Canada Full list of author information is available at the end of the article
}

of patients with bone metastases (Clemons et al. 2012; Coleman 2011; Dougall et al. 2014; Drooger et al. 2013).

Initial studies of bisphosphonates, treated patients every 3-4 weeks concurrently with chemotherapy. However, given the long terminal half life of these agents, and the fact that patients may be treated with them for years, their dosing frequency has come into question (Kimmel 2007), leading to a number of trials looking at de-escalated therapy. To date both single arm (Addison et al. 2014) and randomized trials (Amadori et al. 2013; Hortobagyi et al. 2014; Amir et al. 2013) have confirmed that de-escalated treatment is feasible and at least over the short term is not associated with a greater risk of SREs. It is also noteworthy that cumulative exposure to these drugs is associated with significant toxicities (Mariotti 2008), which could be reduced with use of deescalated treatment strategies. One potential caveat to 
de-escalated regimens is that bone-turnover markers such as N-telopeptide (NTx) (Amadori et al. 2013) or C-telopeptide (CTx) (Amir et al. 2013) which are often used to monitor bone metastases progression appear to rise more substantially in de-escalated arms. These findings raise questions around the long term safety of deescalation of bone targeted therapy.

The REFORM trial was a pilot randomized trial in which breast cancer patients with bone metastases considered to be at "low risk" of complications from their metastases (based on levels of the bone turnover marker CTx $<600 \mathrm{ng} / \mathrm{L}$ ) were randomized to continue therapy once every 3-4 weekly (standard) or to de-escalate to treatment once every 12-weekly for 48 weeks (Amir et al. 2013). The primary analysis comprised changes in serum CTx, however patients were also followed for the occurrence of SREs. While changes in CTx were not associated with SRE risk in the REFORM trial the utility of other putative biomarkers of bone activity as predictors of SREs remain of interest. With availability of longer follow-up data we now explore a series of bone biomarkers including CTx (Lipton et al. 2008; Lipton et al. 2011; Coleman et al. 2005), urinary (u)NTx (Rosen et al. 1994; Gorai et al. 1997; Clemens et al. 1997), bone-specific alkaline phosphatase (BSAP), transforming growth factor (TGF)- $\beta$ (Baselga et al. 2008; Desruisseau et al. 2006), procollagen type 1 amino terminal peptide (P1NP) (Garnero et al. 2000a; Chevrel et al. 2007), activinA (Reis et al. 2002), bone sialoprotein (BSP) (Diel et al. 1999) and pain scores (Harris et al. 2007; Broom et al. 2009) and their association with the incidence of SREs in the REFORM trial.

\section{Materials and methods Patient cohorts}

REFORM was a pilot, randomized, non-inferiority trial designed to explore the effect of de-escalated bisphosphonate therapy on women with breast cancer and bone metastases who were biochemically defined as being at low risk of SREs. Women with histologically proven breast cancer with radiological or biopsy confirmed bone metastases, CTx levels in the low-risk range (defined as serum CTx levels in the lowest tertile $[<600 \mathrm{ng} / \mathrm{L}]$ (Garnero et al. 2003)), and who had received 3-4 weekly anti-bone resorption therapy for a minimum of 3 months were eligible for entry into the REFORM study (Amir et al. 2013). Patients were allocated in an approximate 1:1 fashion to receive pamidronate $90 \mathrm{mg}$ intravenously every 3-4 weeks (control group) or every 12 weeks (de-escalated group) for 48 weeks, stratified according to baseline serum CTx ( $<400 \mathrm{ng} / \mathrm{L}$ and 400-600 ng/L) and duration of prior bisphosphonate use ( $<6$ months and $>6$ months), using a computer-generated permuted blocks design. As part of the main study, serum CTx and bone-specific alkaline phosphatase (BSAP) levels were measured every 12 weeks for
48 weeks. Self-reported pain was also assessed using the validated Brief Pain Inventory (BPI) and Functional Assessment of Cancer Therapy Bone Pain (FACT-BP) questionnaire at baseline and week 12 on study. Patients allocated to the de-escalated treatment group who remained with telopeptide levels below $600 \mathrm{ng} / \mathrm{L}$ continued to receive treatment according to protocoldefined frequency, while patients whose telopeptide levels rose above $600 \mathrm{ng} / \mathrm{L}$ remained on study, but thereafter received treatment every 3-4 weeks. All patients received concomitant vitamin $\mathrm{D}_{3}(800-1000 \mathrm{IU} /$ day) and calcium (1200-1500 mg/day) to prevent hypocalcaemia or secondary hyperparathyroidism. All patients in the main study were asked to participate in this substudy, and only those patients who consented to use of collected specimens for research purposes are included in the present analysis. The study was conducted in accordance with REMARK recommendations for biomarker analyses (McShane et al. 2005), and under institutional ethics approval.

\section{Biochemical analyses}

Serum samples collected after an overnight fast, were allowed to clot for at least 30 minutes then centrifuged at 3,000 rpm for 10 minutes at room temperature. Samples were then processed immediately and stored at $-80^{\circ} \mathrm{C}$. Serum CTx was measured in a central lab facility with an enzyme-linked immunosorbent assay (ELISA; Beta-Cross Laps/serum assay, Roche Diagnostics Canada Inc, Laval, QC; detection limit $10 \mathrm{ng} / \mathrm{L}$, interassay variability, $\sim 6.7 \%)$. Serum BSAP was also measured in a central lab facility by ELISA (Metra Biosystems, San Diego, CA; detection limit 0.7 IU/L, interassay variability $\sim 5.2 \%$ ). Experimental determination of serum levels of TGF- $\beta$ (Quantikine, R\&D Systems, Minneapolis MN, detection limit $20 \mathrm{pg} / \mathrm{ml}$, interassay variability $\sim 8.3 \%$ ), activinA (Quantikine, R\&D Systems, Minneapolis MN, detection limit $4 \mathrm{pg} / \mathrm{ml}$, interassay variability 5.9\%), P1NP (USCN Life Science Inc., Wuhan China, detection limit $15 \mathrm{pg} / \mathrm{ml}$, interassay variability 12\%), and BSP (Cusabio Biotech Co., Wuhan China, detection limit $2 \mathrm{ng} / \mathrm{ml}$, interassay variability $\sim 12 \%$ ) were also experimentally measured by ELISA. Urine NTx levels were measured using the Osteomark assay system (Alere, Scarborough ME, detection limit $2 \mathrm{nM} \mathrm{BCE} / \mathrm{mM}$ creatine, interassay variability $\sim 6.9 \%$ ). All samples were measured in duplicate and concentrations determined following interpolation of standard curves generated from known quantities of recombinant protein. When values were below the threshold of detection for each respective assay, concentrations were assigned as 0.1 below the sensitivity threshold for purposes of statistical analysis. 


\section{Statistical analysis}

Descriptive statistics were used to summarize patient characteristics and laboratory measurements across time points. Spearman correlation coefficients were used to investigate for the potential of intra-biomarker variability and to examine potential relationships between different biomarkers at each time point. Univariable logistic regression analysis was conducted to determine the association between selected biomarkers and clinical characteristics and SREs. Multivariable analysis was not conducted due to the small sample size. The nonparametric Mann-Whitney $U$ test was used to test for differences in biomarkers at different time points between cohorts. Statistical significance was defined as a p-value of 0.05 or less, and all tests were two-sided. As this study is exploratory no adjustment for multiple testing was made and validation of results from additional studies are required.

\section{Ethical standards}

This study was performed in accordance with ethical standards approved by the Ottawa Health Science Network Research Ethics Board (20120403-01H) and the University Health Network (08-0513-C).

\section{Results}

\section{Patient cohorts}

Thirty of the 38 patients (79\%) who were randomized to the original study provided consent for this substudy (see Figure 1). Of these 30 patients, 13 were randomized to Group 1 (q3-4 weekly) and 17 to Group 2 (12 weekly). Samples were available at week 12 post study entry for 10 patients from Group 1 and 13 from Group 2. Patients were followed for 2 years (one year on study and one additional year of follow up for which frequency of pamidronate use was not specified) during which time seven SREs were observed among the 25

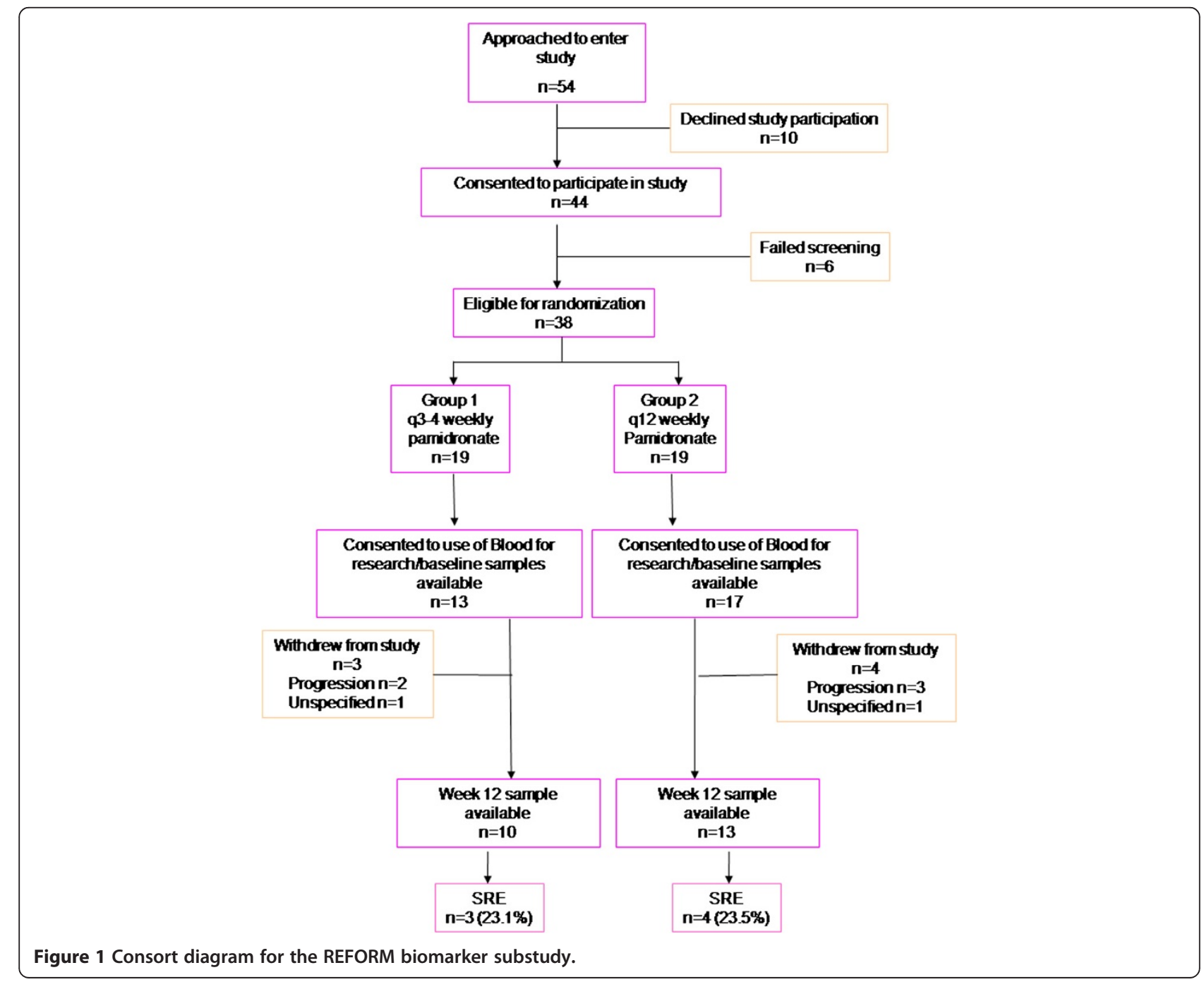


patients with complete data; 3 patients in the control group and 4 patients in the experimental group.

\section{Biomarker correlations}

As each biomarker was measured in duplicate, the intrabiomarker variability was evaluated. The Spearman correlation coefficient exceeded $\rho=0.92$ between duplicated measurements for each biomarker at each time point, indicating little to no intra-biomarker variability. Thus, the addition of the duplicate measure added little to no additional information, and as a result, only the first biomarker value is discussed for all further analyses.

The association between different biomarkers at baseline and at week 12, the association of baseline and week 12 biomarker values, as well as the association of the change from baseline to week 12 between different biomarkers, are presented in Additional files 1 and 2: Tables S1 (baseline) and S2 (week 12) respectively. Generally, only weak to moderate correlations were observed between the different biomarker measures. Baseline CTx and BSAP were strongly associated with week 12 measures, while baseline NTx, P1NP, TGF- $\beta$ and activin-A were all moderately associated for their values at week 12 .

Biomarker values at baseline and at week 12 were generally similar between the two treatment arms (Table 1). As can be seen in Table 2, those patients with higher levels of baseline CTx $(p=0.002), \operatorname{BSAP}(p=0.005)$, P1NP ( $p=0.002)$ and ActivinA $(\mathrm{p}<0.001)$ were significantly more likely to not complete week 12 of the study. It is noted that CTx $>600 \mathrm{ng} / \mathrm{L}$ was a trial mandated reason for coming off study early.
The change in biomarker levels from baseline to week 12 on study was assessed, given that week 12 levels are often used in clinical trials as a surrogate marker of bone metastasis control and hence bisphosphonate efficacy (Lipton et al. 2008; Coleman et al. 2005; Brown et al. 2003; Lipton et al. 1998). Significant increases in biomarker levels from baseline to week 12 for CTx $(\mathrm{p}<$ $0.001)$, BSAP $(p=0.011)$ and activinA $(p=0.001)$ were noted for the entire cohort of patients i.e. whether or not they were randomized to de-escalated therapy (Table 3). Comparing between treatment arms, patients in treatment group 2 (q12 weekly arm) had statistically significantly greater increases in CTx (median of 131 versus $17, \mathrm{p}=0.034$ ) and in BSAP (median $=3$ versus 0 , $\mathrm{p}=0.010)$.

\section{Association between biomarkers and pain}

Baseline pain scores tended to be higher in patients randomized to 12-weekly therapy ( $\mathrm{p}=0.051$ for FACT-BP and $\mathrm{p}=0.12$ for $\mathrm{BPI})$, but pain scores were similar at the week 12 time point $(\mathrm{p}=0.64$ for FACT-BP and $\mathrm{p}=0.55$ for BPI). Interestingly, pain scores as measured by both FACT-BP $(\mathrm{p}=0.053)$ and BPI $(\mathrm{p}=0.050)$ tended to be more reduced from baseline to 12 weeks in the patients treated with the de-escalated regimen as compared to 3-4 weekly treated patients (Table 3), although it is noted that the p-value was low and did not attain the pre-defined level of statistical significance.

\section{Association between biomarkers and SRE incidence}

Results from univariable exploratory analyses to determine whether circulating levels of biomarkers were significantly

Table 1 Biomarker and clinical characteristics

\begin{tabular}{|c|c|c|c|c|c|c|c|}
\hline & & \multicolumn{3}{|c|}{ Baseline } & \multicolumn{3}{|c|}{ Week 12} \\
\hline & & All patients & Group 1 & Group 2 & All patients & $\begin{array}{l}\text { Treatment } \\
\text { group } 1\end{array}$ & $\begin{array}{l}\text { Treatment } \\
\text { group } 2\end{array}$ \\
\hline $\mathbf{N}$ & & 30 & 13 & 17 & 23 & 10 & 13 \\
\hline CTx ng/L & Median (IQR) & $191(104,387)$ & $142(117,287)$ & $218(88,387)$ & $223(106,381)$ & $155(106,225)$ & $308(179,423)$ \\
\hline BSAP IU/L & Median (IQR) & $18(14,30)$ & $16(14,30)$ & $18(14,29)$ & $17(14,29)$ & $16(12,29)$ & $18(16,25)$ \\
\hline $\mathrm{TGF}-\beta, \mathrm{ng} / \mathrm{ml}$ & Median (IQR) & $22.5(17.1,26.3)$ & $24.8(20.7,26.3)$ & $20.3(17.1,25.5)$ & $21.5(15.8,26.6)$ & $21.7(15.0,26.6)$ & $21.5(18.0,25.7)$ \\
\hline Activin-A, pg/ml & Median (IQR) & $597(419,1528)$ & $795(517,1164)$ & $519(404,1666)$ & $560(483,1045)$ & $616(483,1153)$ & $560(526,755)$ \\
\hline NTx 2nM BCE/mM creatine & Median (IQR) & $158(73,282)$ & $159(60,298)$ & $149(87,268)$ & $258(121,338)$ & $299(94,338)$ & $230(137,342)$ \\
\hline P1NP ng/ml & Median (IQR) & $56(48,91)$ & $70(48,96)$ & $55(4,80)$ & $54(38,75)$ & $55(36,75)$ & $54(52,60)$ \\
\hline BSP ng/ml & Median (IQR) & $34(13,60)$ & $39(10,73)$ & $32(16,55)$ & $17(5,21)$ & $18(16,21)$ & $14(5,36)$ \\
\hline FACT-BP & Median (IQR) & $13(5,25)$ & $8(5,14)$ & $16(11,26)$ & $13.5(8,18)$ & $11(8,17)$ & $14(11,18)$ \\
\hline BPI & Median (IQR) & $16(0,32)$ & $4(0,29)$ & $19(6,57)$ & $14.5(2,21)$ & $13(1,20)$ & $15(7,21)$ \\
\hline \multirow{4}{*}{$\begin{array}{l}\text { \# of SRE }(\%), \text { on or } \\
\text { post study }(n=25)\end{array}$} & 0 & $11(44.0)$ & $5(45.5)$ & $6(42.9)$ & $18(72.0)$ & $8(72.7)$ & $10(71.4)$ \\
\hline & 1 & $9(36.0)$ & $3(27.7)$ & $6(42.9)$ & $3(12.0)$ & $2(18.2)$ & $1(7.1)$ \\
\hline & 2 & $5(20.0)$ & $3(27.7)$ & $2(14.3)$ & $3(12.0)$ & $1(9.1)$ & $2(14.3)$ \\
\hline & 3 & & & & $1(4.0)$ & $0(0.0)$ & $1(7.1)$ \\
\hline
\end{tabular}


Table 2 Factors associated with failure to complete 12 weeks on study

\begin{tabular}{|c|c|c|c|c|}
\hline & & Did not complete week 12 & Completed week 12 & p-value \\
\hline $\mathbf{N}$ & & 7 & 23 & \\
\hline CTx & Median (IQR) & $656(492,811)$ & $141(88,229)$ & 0.002 \\
\hline BSAP & Median (IQR) & $44(26,68)$ & $15(14,22)$ & 0.005 \\
\hline TGF- $\beta, \mathbf{n g} / \mathrm{ml}$ & Median (IQR) & $26.3(16.8,30.7)$ & $20.7(17.1,25.5)$ & 0.38 \\
\hline Activin-A, pg/ml & Median (IQR) & $2922(1666,9576)$ & $517(404,775)$ & $<0.001$ \\
\hline NTx & Median (IQR) & NA & $158(73,282)$ & - \\
\hline P1NP & Median (IQR) & $123075(80014,178930)$ & $54334(36692,77623)$ & 0.002 \\
\hline BSP & Median (IQR) & $63.9(55.3,90.4)$ & $15.9(8.2,32.9)$ & 0.004 \\
\hline FACT-BP & Median (IQR) & $15(7,36)$ & $12(5,23)$ & 0.37 \\
\hline BPI & Median (IQR) & $57(12,65)$ & $6(0,29)$ & 0.049 \\
\hline \multirow[t]{3}{*}{ \# of SRE, pre, study $(n=25)$} & 0 & 1 & 10 & \\
\hline & 1 & 0 & 9 & \\
\hline & 2 & 1 & 4 & \\
\hline \multirow[t]{4}{*}{ \# of SRE, on or post, study $(n=25)$} & 0 & 2 & 16 & \\
\hline & 1 & 0 & 3 & \\
\hline & 2 & 0 & 3 & \\
\hline & 3 & 0 & 1 & \\
\hline Duration of bone mets, in months & Median (IQR) & $28(18,41)$ & $18(12,29)$ & 0.19 \\
\hline \multirow[t]{3}{*}{ Time from primary to bone mets $(n=25)$} & $<2$ years & 1 & 12 & \\
\hline & $2-5$ years & 0 & 5 & \\
\hline & $>5$ years & 1 & 6 & \\
\hline
\end{tabular}

different between patients who experienced on study SREs versus those that did not are shown in Table 4. Interestingly, 6/14 (43\%) patients who had previous SREs prior to study entry, had an on study SRE, while only 1/11 (9\%) without a prior SRE experienced an on study SRE (p-value $=0.088$ ). At 2 years of follow up, SRE rates remained similar between the two cohorts, with $3 / 11(27 \%)$ of 3-4 weekly treated patients and 4/14 (29\%) of 12 weekly treated patients having experienced SREs $(\mathrm{p}$-value $=0.94)$.
No significant association was observed for any of the baseline biomarkers, including the more commonly used markers such as CTx and NTx (Table 4), as prognostic factors for experiencing a SRE. Similarly, the change in level from baseline to week 12 was not significantly associated with experiencing on study SREs for any biomarker (Table 4). Of all the parameters measured, only baseline pain as measured by FACT-BP was statistically significantly associated with development of an on or post-study SRE $(\mathrm{p}=0.041$, Table 4$)$. Changes in pain

Table 3 Changes in biomarker levels from baseline to 12-weeks on treatment

\begin{tabular}{|c|c|c|c|c|c|c|}
\hline & & Difference all patients & $\mathrm{p}$-value & $\begin{array}{c}\text { Difference treatment } \\
\text { group } 1\end{array}$ & $\begin{array}{c}\text { Difference treatment } \\
\text { group } 2\end{array}$ & p-value \\
\hline $\mathrm{N}$ & & 23 & & 10 & 13 & \\
\hline CTx ng/L & Median (IQR) & $83(2,155)$ & $<0.001$ & $17(-27,83)$ & $131(79,171)$ & 0.034 \\
\hline BSAP IU/L & Median (IQR) & $1(0,4)$ & 0.011 & $0(-1,1)$ & $3(2,5)$ & 0.010 \\
\hline TGF- $\beta, \mathrm{ng} / \mathrm{ml}$ & Median (IQR) & $0.8(-2.1,4.9)$ & 0.28 & $-0.1(-2.1,2.2)$ & $2.4(-0.0,4.9)$ & 0.41 \\
\hline Activin-A, pg/ml & Median (IQR) & $111(28,526)$ & 0.001 & $96(-29,596)$ & $122(34,256)$ & 0.60 \\
\hline NTx 2nM BCE/mM creatine & Median (IQR) & $42(-48,168)$ & 0.15 & $42(-15,179)$ & $45(-76,108)$ & 0.54 \\
\hline P1NP ng/ml & Median (IQR) & $5742(-6694,19129)$ & 0.24 & $1376(-6694,8498)$ & $16539(-1266,21993)$ & 0.26 \\
\hline BSP $\mathrm{ng} / \mathrm{ml}$ & Median (IQR) & $5.8(-8.0,9.5)$ & 0.63 & $0.5(-15.4,6.4)$ & $9.8(0.8,47.2)$ & 0.14 \\
\hline FACT-BP & Median (IQR) & $0(-4,3)$ & 0.99 & $1(0,4)$ & $-2(-4,1)$ & 0.053 \\
\hline BPI & Median (IQR) & $0(-3,4)$ & 0.94 & $2(0,7)$ & $-1(-8,1)$ & 0.050 \\
\hline
\end{tabular}


Table 4 Association of biomarkers with odds of having an on or post-study SRE

\begin{tabular}{|c|c|c|c|c|c|}
\hline & & \multicolumn{2}{|l|}{ Baseline } & \multicolumn{2}{|c|}{ Change from Baseline to 12 Weeks } \\
\hline & & Odds ratio $(95 \% \mathrm{Cl})$ & p-value & Odds ratio $(95 \% \mathrm{Cl})$ & p-value \\
\hline CTx & $/ 10$ unit change & $1.00(0.94,1.07)$ & 0.99 & $1.04(0.95,1.14)$ & 0.39 \\
\hline BSAP & /unit change & $1.03(0.97,1.09)$ & 0.36 & $0.90(0.73,1.11)$ & 0.32 \\
\hline TGF- $\beta$ & /unit change & $1.05(0.90,1.23)$ & 0.54 & $0.98(0.83,1.16)$ & 0.83 \\
\hline Activin-A & $/ 100$ unit change & $0.98(0.89,1.07)$ & 0.60 & $0.99(0.93,1.04)$ & 0.64 \\
\hline NTx & $/ 10$ unit change & $1.00(0.97,1.02)$ & 0.69 & $1.01(0.97,1.06)$ & 0.56 \\
\hline P1NP & $/ 10000$ unit change & $0.97(0.79,1.18)$ & 0.75 & $0.98(0.80,1.20)$ & 0.84 \\
\hline BSP & $/ 10$ unit change & $1.00(0.63,1.60)$ & 0.99 & $0.94(0.60,1.47)$ & 0.79 \\
\hline FACT-BP & /unit change & $1.13(1.01,1.28)$ & 0.041 & $0.99(0.87,1.14)$ & 0.93 \\
\hline BPI & /unit change & $1.03(0.99,1.08)$ & 0.16 & $1.00(0.94,1.06)$ & 0.93 \\
\hline Duration of bone metastases & /month & $1.03(0.99,1.08)$ & 0.14 & & \\
\hline Pre-Study SRE & Yes versus no & $7.50(0.74,75.72)$ & 0.088 & & \\
\hline Treatment Group & 2 versus 1 & $1.07(0.18,6.21)$ & 0.94 & & \\
\hline
\end{tabular}

levels from baseline to 12 weeks, as measured by FACTBP, were not associated with SRE development (Table 4).

\section{Discussion}

De-escalated bisphosphonate therapy is being increasingly utilized after data from the large randomized ZOOM and OPTIMIZE-2 trials showed that skeletal outcomes are similar to those of patients receiving standard 3-4 weekly treatment (Amadori et al. 2013; Hortobagyi et al. 2014). As confirmed in our present study, a common feature of studies of de-escalated bisphosphonate therapy appears to be modest increases in telopeptide levels from baseline to week 12 in the deescalated arm, while levels are maintained in the standard frequency arm. Despite this, no differences in either the number of SREs (REFORM (Amir et al. 2013) and current study), time to first on-study SRE (ZOOM (Amadori et al. 2013)) or SRE rate (OPTIMIZE-2 (Hortobagyi et al. 2014)) were observed between the two treatment groups. This raises the question of whether or not circulating telopeptide levels on treatment are truly related with SRE risk. With the continuing trend of clinical studies being designed to use measurement of these markers as endpoints to determine drug efficacy, and the fact that patients on these drugs experience SREs regardless of the level of telopeptides, it is of the utmost importance to gain a better understanding of the relationship between biomarker measures and subsequent SRE risk.

In the current analysis a range of recognized boneturnover or metastasis biomarkers were measured. Urinary $\mathrm{N}$-telopeptide (uNTx) is a fragment of collagen I produced during osteolysis of the bone and has frequently been used to monitor bone resorption in both osteoporotic (Yoshimura et al. 2011; Garnero 2008; Reginster et al. 2001) and bone metastases (Lipton et al. 2008; Lipton et al. 2011; Coleman et al. 2005) patients. Elevated levels of uNTx have been shown to correlate with bone turnover (Rosen et al. 1994; Gorai et al. 1997; Clemens et al. 1997). P1NP is a marker of bone formation (Garnero et al. 2000b) which has been shown to correlate with presence of bone metastases in prostate cancer patients (Garnero et al. 2000a), and previously was shown to be associated with increased bone fractures (Chevrel et al. 2007). TGF- $\beta$ plays a critical role in the exacerbation of osteolytic metastatic disease by contributing to continued osteoclast activity and aggressive and invasive tumour phenotypes (Ivanovic et al. 2003). Furthermore, plasma TGF- $\beta$ levels have been found to correlate with disease stage and bone metastasis in breast cancer (Baselga et al. 2008; Desruisseau et al. 2006). Similarly recent studies have suggested that the TGF- $\beta$ family member, activinA, also plays a role in metastatic and osteoclastic processes (Leto et al. 2006; Risbridger et al. 2001; Fuller et al. 2000). Plasma levels of activinA were shown to be higher in breast cancer patients with bone metastases as compared to those without and to positively correlate with the number of bone metastases (Reis et al. 2002). Lastly, tumour-derived expression of BSP also correlates with subsequent development of osseous metastases, as it has been shown that baseline serum BSP levels were elevated in $\sim 90 \%$ of women who went on to develop bone metastases, while not elevated in patients who developed visceral metastases (Diel et al. 1999).

None of the measured parameters at baseline nor their changes from baseline to week 12, were observed to predict for subsequent SREs. The fact that other more routinely used markers such as CTx and UNTx were not significantly different in patients who experienced SRE versus those that did not in our study may simply be a result of the fact that these cohorts were too small to 
discern those differences. Although previous studies have suggested that median levels of many bone turnover biomarkers tend to be higher in the patients who experienced SREs (Coleman et al. 2008), additional studies lead us to question this notion in bone metastatic patients considered to be low risk due to bone turnover markers closer to the 'normal range'. Recent results with NTx have suggested that in patients with uNTx levels considered to be in the low range, $>90 \%$ of the patients who experienced SREs had no documented increase in NTx prior to their SRE (Lipton et al. 2013). A further possibility is that in patients with low baseline levels of telopeptides changes in these markers are not predictive of bone outcomes. It remains unclear if this observation can be extrapolated to patients with higher baseline biomarker levels.

Of all the parameters measured in the current study, it is interesting that pain at baseline was prognostic for occurrence of subsequent SREs. Previous analyses in metastatic breast cancer patients treated with the bisphosphonate zoledronic acid, also found that baseline pain was associated with time to first SRE in univariable analysis (Brown et al. 2010). However, in this study, pain was not associated with skeletal fractures, but was significantly associated with need for radiation treatment. Of the 7 patients who experienced SREs in REFORM all required radiotherapy (although each may have had additional SREs of other types in conjunction), which may explain the observed association between baseline pain and occurrence of SREs. Despite this, given that pain significantly affects a patient's quality of life and is associated with need for radiation treatment for skeletal complications, its measurement in future studies is warranted.

With data suggesting that de-escalation of bisphosphonate therapy may be equally therapeutically effective yet also spare patients exposure to the detrimental effects of excessive cumulative dose of bisphosphonates (Amadori et al. 2013; Amir et al. 2012), it is essential to better understand the pharmacodynamics of bone turnover markers in response to these agents if clinical decisions are to be made based on these factors. While markers such as NTx and CTx may be very effective as prognostic indicators of bone turnover in patients with bone metastases starting bone targeted therapies, additional markers assessing risk of SRE may be required. This may be particularly important in those cases where patients are found to have NTx and CTx levels considered to be in the "low" or "normal" range with bone-targeted treatments. Furthermore, it is unclear what effects concomitant anti-cancer therapy may have on the relative levels of these markers that may further confound their utility as predictive markers on treatment. Recent studies have shown that use of exemestane results in increased levels from baseline for BSAP, P1NP and CTx, while addition of everolimus to exemestane resulted in decreased levels of the same biomarkers after baseline (Gnant et al. 2013). Importantly, the changes in biomarker levels were observed in patients irrespective of the presence of bone metastases, highlighting the effect of chemotherapy on normal bone homeostasis in general. Interestingly, a large recent biomarker driven study in patients starting fulvestrant also showed significant decreases in uNTx from baseline to on treatment (Clemons et al. 2014). As these patients had no change in their bone-targeted therapy at the time of study entry, this confirms that changes in anticancer therapies can alter bone biomarker levels.

This study has a number of limitations. It was specifically designed as a small study to assess the feasibility of randomizing patients to de-escalated therapy. As such it has a small cohort and even with the extended follow up, a low number of SREs has occurred. Furthermore, 5 patients were lost to follow up over the year following the end of the REFORM study, further restricting our sample size. It is also of interest to note that only pain as assessed by FACT-BP but not by BPI was associated with SRE occurrence, despite both tests being validated measurement tools. The reasons for this remain unclear. Finally, all patients enrolled in our study had low baseline levels of CTx and therefore, our results may not be generalizable to patients with bone metastases who present with a greater magnitude of bone resorption.

\section{Conclusions}

In conclusion, these findings show that no significant changes in any of the biomarkers tested were observed between the standard and the de-escalated treatment groups. Exploratory analysis suggested that biomarkers of bone activity do not appear to predict for SREs in the 'low risk' bone metastases patients. Bone pain does however appear to be associated with SRE occurrence, a finding which warrants evaluation in larger studies.

\section{Additional files}

Additional file 1: Table S1. Spearman Correlation of Baseline Biomarkers.
Additional file 2: Table S2. Spearman Correlation of Change in Biomarkers
from Baseline to Week 12 .

Abbreviations

BPI: Brief pain index; BSAP: Bone specific alkaline phosphatase; BSP: Bone sialoprotein; CTx: C-telopeptide; FACT-BP: Functional assessment of cancer therapy-bone pain; TGF- $\beta$ : Transforming growth factor beta; NTx: N-telopeptide; P1NP: Pro-collagen type 1 amino terminal peptide; SRE: Skeletal related event.

Competing interests

The authors declare that they have no competing interests.

\section{Authors' contributions}

The current study was designed and overseen by CLA, who also was responsible for data compilation, verification and analysis. MC, and EA were 
involved in the patient enrollment and conducting of the REFORM clinical trial. Clinical outcome data was collected by MC, EA, LV and RG. ELISA analysis was performed by $\mathrm{HZ}$ and $\mathrm{SM}$. All statistical analyses were performed by GP. Manuscript drafting was performed by CLA and MC, with editing contributions by all remaining authors. All authors read and approved the final manuscript.

\section{Acknowledgements}

The authors would like to acknowledge the generous support of this study through funding from the Ottawa Hospital Foundation. No pharmaceutical sponsorship was obtained for the present study. We would like to also thank Koralee Berghout for assistance with REB approvals.

\section{Author details}

'Program for Cancer Therapeutics, Ottawa Hospital Research Institute, Ottawa, ON, Canada. ${ }^{2}$ Department of Medicine, University of Ottawa, Ottawa, ON, Canada. ${ }^{3}$ Biochemistry, Microbiology and Immunology, University of Ottawa, Ottawa, ON, Canada. ${ }^{4}$ Department of Oncology, McMaster University, Hamilton, Canada. ${ }^{5}$ Princess Margaret Hospital, Toronto, ON, Canada.

\section{Received: 8 September 2014 Accepted: 9 September 2014} Published: 1 October 2014

\section{References}

Addison CL, Bouganim N, Hilton J, Vandermeer L, Dent S, Amir E, Hopkins S, Kuchuk I, Segal R, Song X, Gertler S, Mazzarello S, Dranitsaris G, Ooi D, Pond G, Clemons M (2014) A phase II, multicentre trial evaluating the efficacy of de-escalated bisphosphonate therapy in metastatic breast cancer patients at low-risk of skeletal-related events. Breast Cancer Res Treat 144(3):615-624, doi:10.1007/s10549-014-2906-X

Amadori D, Aglietta M, Alessi B, Gianni L, Ibrahim T, Farina G, Gaion F, Bertoldo F, Santini D, Rondena R, Bogani P, Ripamonti Cl (2013) Efficacy and safety of 12-weekly versus 4-weekly zoledronic acid for prolonged treatment of patients with bone metastases from breast cancer (ZOOM): a phase 3, open-label, randomised, non-inferiority trial. Lancet Oncol 14(7):663-670, doi:10.1016/S1470-2045(13)70174-8

Amir E, Freedman O, Carlsson L, Dranitsaris G, Tomlinson G, Laupacis A, Tannock IF, Clemons M (2012) Randomized Feasibility Study of De-escalated (Every 12 wk) Versus Standard (Every 3 to $4 \mathrm{wk}$ ) Intravenous Pamidronate in Women With Low-risk Bone Metastases From Breast Cancer. Am J Clin Oncol, doi:10.1097/COC.0b013e3182568f7a

Amir E, Freedman O, Carlsson L, Dranitsaris G, Tomlinson G, Laupacis A, Tannock IF, Clemons M (2013) Randomized feasibility study of de-escalated (every 12 wk) versus standard (every 3 to $4 \mathrm{wk}$ ) intravenous pamidronate in women with low-risk bone metastases from breast cancer. Am J Clin Oncol 36 (5):436-442, doi:10.1097/COC.0b013e3182568f7a

Baselga J, Rothenberg ML, Tabernero J, Seoane J, Daly T, Cleverly A, Berry B, Rhoades SK, Ray CA, Fill J, Farrington DL, Wallace LA, Yingling JM, Lahn M, Arteaga C, Carducci M (2008) TGF-beta signalling-related markers in cancer patients with bone metastasis. Biomarkers 13(2):217-236, doi:10.1080/ 13547500701676019

Broom R, Du H, Clemons M, Eton D, Dranitsaris G, Simmons C, Ooi W, Cella D (2009) Switching breast cancer patients with progressive bone metastases to third-generation bisphosphonates: measuring impact using the Functional Assessment of Cancer Therapy-Bone Pain. J Pain Symptom Manage 38(2):244-257, doi:10.1016/j.jpainsymman.2008.08.005

Brown JE, Thomson CS, Ellis SP, Gutcher SA, Purohit OP, Coleman RE (2003) Bone resorption predicts for skeletal complications in metastatic bone disease. $\mathrm{Br} \mathrm{J}$ Cancer 89(11):2031-2037, doi:10.1038/s.bj..6601437

Brown JE, Cook RJ, Lipton A, Costa L, Coleman RE (2010) Prognostic factors for skeletal complications from metastatic bone disease in breast cancer. Breast Cancer Res Treat 123(3):767-779, doi:10.1007/s10549-010-0981-1

Chevrel G, Limouzin A, Garnero P, De Montalivet C, Loubier D (2007) Fractures and handicap in an adult population: A clinical study. Joint Bone Spine 74 (6):584-589, doi:10.1016/j.jbspin.2007.01.039

Clemens JD, Herrick MV, Singer FR, Eyre DR (1997) Evidence that serum NTx (collagen-type I N-telopeptides) can act as an immunochemical marker of bone resorption. Clin Chem 43(11):2058-2063

Clemons M, Gelmon KA, Pritchard KI, Paterson AH (2012) Bone-targeted agents and skeletal-related events in breast cancer patients with bone metastases: the state of the art. Curr Oncol 19(5):259-268, doi:10.3747/co.19.1011
Clemons MJ, Cochrane B, Pond GR, Califaretti N, Chia SK, Dent RA, Song X, Robidoux A, Parpia S, Warr D, Rayson D, Pritchard Kl, Levine MN (2014) Randomised, phase II, placebo-controlled, trial of fulvestrant plus vandetanib in postmenopausal women with bone only or bone predominant, hormone-receptor-positive metastatic breast cancer (MBC): the OCOG ZAMBONEY study. Breast Cancer Res Treat 146(1):153-162, doi:10.1007/ s10549-014-3015-6

Coleman R (2011) The use of bisphosphonates in cancer treatment. Ann N Y Acad Sci 1218:3-14, doi:10.1111/j.1749-6632.2010.05766.x

Coleman RE, Rubens RD (1987) The clinical course of bone metastases from breast cancer. Br J Cancer 55(1):61-66

Coleman RE, Major P, Lipton A, Brown JE, Lee KA, Smith M, Saad F, Zheng M, Hei YJ, Seaman J, Cook R (2005) Predictive value of bone resorption and formation markers in cancer patients with bone metastases receiving the bisphosphonate zoledronic acid. J Clin Oncol 23(22):4925-4935, doi:10.1200/JCO.2005.06.091

Coleman R, Brown J, Terpos E, Lipton A, Smith MR, Cook R, Major P (2008) Bone markers and their prognostic value in metastatic bone disease: clinical evidence and future directions. Cancer Treat Rev 34(7):629-639, doi:10.1016/j.ctrv.2008.05.001

Desruisseau S, Palmari J, Giusti C, Romain S, Martin PM, Berthois Y (2006) Determination of TGFbeta1 protein level in human primary breast cancers and its relationship with survival. Br J Cancer 94(2):239-246, doi:10.1038/s..bjc.6602920

Diel IJ, Solomayer EF, Seibel MJ, Pfeilschifter J, Maisenbacher H, Gollan C, Pecherstorfer M, Conradi R, Kehr G, Boehm E, Armbruster FP, Bastert G (1999) Serum bone sialoprotein in patients with primary breast cancer is a prognostic marker for subsequent bone metastasis. Clin Cancer Res 5 (12):3914-3919

Dougall WC, Holen I, Gonzalez Suarez E (2014) Targeting RANKL in metastasis. Bonekey Rep 3:519, doi:10.1038/bonekey.2014.14

Drooger JC, van der Padt A, Sleijfer S, Jager A (2013) Denosumab in breast cancer treatment. Eur J Pharmacol 717(1-3):12-19, doi:10.1016/j. ejphar.2013.03.034

Fuller K, Bayley KE, Chambers TJ (2000) Activin A is an essential cofactor for osteoclast induction. Biochem Biophys Res Commun 268(1):2-7, doi:10.1006/ bbrc. 2000.2075

Garnero P (2008) Biomarkers for osteoporosis management: utility in diagnosis, fracture risk prediction and therapy monitoring. Mol Diagn Ther 12(3):157-170

Garnero P, Buchs N, Zekri J, Rizzoli R, Coleman RE, Delmas PD (2000a) Markers of bone turnover for the management of patients with bone metastases from prostate cancer. Br J Cancer 82(4):858-864, doi:10.1054/bjoc.1999.1012

Garnero P, Rousseau JC, Delmas PD (2000b) Molecular basis and clinical use of biochemical markers of bone, cartilage, and synovium in joint diseases. Arthritis Rheum 43(5):953-968, doi:10.1002/1529-0131(200005)43:5<953:-AID-ANR1>3.0. $\mathrm{CO} ; 2-\mathrm{Q}$

Garnero P, Mulleman D, Munoz F, Sornay-Rendu E, Delmas PD (2003) Long-term variability of markers of bone turnover in postmenopausal women and implications for their clinical use: the OFELY study. J Bone Miner Res 18 (10):1789-1794, doi:10.1359/jbmr.2003.18.10.1789

Gnant M, Baselga J, Rugo HS, Noguchi S, Burris HA, Piccart M, Hortobagyi GN, Eakle J, Mukai H, Iwata H, Geberth M, Hart LL, Hadji P, El-Hashimy M, Rao S, Taran T, Sahmoud T, Lebwohl D, Campone M, Pritchard KI (2013) Effect of everolimus on bone marker levels and progressive disease in bone in BOLERO-2. J Natl Cancer Inst 105(9):654-663, doi:10.1093/jnci/djt026

Gorai I, Taguchi Y, Chaki O, Nakayama M, Minaguchi H (1997) Specific changes of urinary excretion of cross-linked $\mathrm{N}$-telopeptides of type I collagen in pre- and postmenopausal women: correlation with other markers of bone turnover. Calcif Tissue Int 60(4):317-322

Harris K, Li K, Flynn C, Chow E (2007) Worst, average or current pain in the Brief Pain Inventory: which should be used to calculate the response to palliative radiotherapy in patients with bone metastases? Clin Oncol (R Coll Radiol) 19 (7):523-527, doi:10.1016/j.clon.2007.04.007

Hortobagyi GN, Lipton A, Chew HK, Gradishar WJ, Sauter NP, Mohanlal RW Zheng M, McGrain B, Van Poznak C (2014) Efficacy and safety of continued zoledronic acid every 4 weeks versus every 12 weeks in women with bone metastases from breast cancer: Results of the OPTIMIZE-2 trial. In: Oncologists ASOC (ed) 2014 ASCO Annual Meeting. vol 5S. Journal of Clinical Oncology, p LBA9500, Chicago, IL

Ivanovic V, Todorovic-Rakovic N, Demajo M, Neskovic-Konstantinovic Z, Subota V, Ivanisevic-Milovanovic O, Nikolic-Vukosavljevic D (2003) Elevated plasma 
levels of transforming growth factor-beta 1 (TGF-beta 1) in patients with advanced breast cancer: association with disease progression. Eur J Cancer 39(4):454-461

Kimmel DB (2007) Mechanism of action, pharmacokinetic and pharmacodynamic profile, and clinical applications of nitrogen-containing bisphosphonates. J Dent Res 86(11):1022-1033

Leto G, Incorvaia L, Badalamenti G, Tumminello FM, Gebbia N, Flandina C, Crescimanno M, Rini G (2006) Activin A circulating levels in patients with bone metastasis from breast or prostate cancer. Clin Exp Metastasis 23 (2):117-122, doi:10.1007/s10585-006-9010-5

Lipton A, Demers L, Curley E, Chinchilli V, Gaydos L, Hortobagyi G, Theriault R, Clemens D, Costa L, Seaman J, Knight R (1998) Markers of bone resorption in patients treated with pamidronate. Eur J Cancer 34(13):2021-2026

Lipton A, Cook R, Saad F, Major P, Garnero P, Terpos E, Brown JE, Coleman RE (2008) Normalization of bone markers is associated with improved survival in patients with bone metastases from solid tumors and elevated bone resorption receiving zoledronic acid. Cancer 113(1):193-201, doi:10.1002/ cncr.23529

Lipton A, Costa L, Coleman RE (2011) Bone turnover markers: tools for prognosis and monitoring response to bisphosphonates? Breast Dis 33(2):59-69, doi:10.3233/BD-2010-0327

Lipton A, Cook R, Brown J, Body JJ, Smith M, Coleman R (2013) Skeletal-related events and clinical outcomes in patients with bone metastases and normal levels of osteolysis: exploratory analyses. Clin Oncol (R Coll Radiol) 25(4):217-226, doi:10.1016/j.clon.2012.11.004

Mariotti A (2008) Bisphosphonates and osteonecrosis of the jaws. J Dent Educ 72 (8):919-929

McShane LM, Altman DG, Sauerbrei W, Taube SE, Gion M, Clark GM (2005) Reporting recommendations for tumor marker prognostic studies (REMARK). J Natl Cancer Inst 97(16):1180-1184

Reginster JY, Henrotin Y, Christiansen C, Gamwell-Henriksen E, Bruyere CJ, Christgau S (2001) Bone resorption in post-menopausal women with normal and low BMD assessed with biochemical markers specific for telopeptide derived degradation products of collagen type I. Calcif Tissue Int 69(3):130-137

Reis FM, Cobellis L, Tameirao LC, Anania G, Luisi S, Silva IS, Gioffre W, Di Blasio AM, Petraglia $F$ (2002) Serum and tissue expression of activin a in postmenopausal women with breast cancer. J Clin Endocrinol Metab 87 (5):2277-2282

Risbridger GP, Schmitt JF, Robertson DM (2001) Activins and inhibins in endocrine and other tumors. Endocr Rev 22(6):836-858

Rosen HN, Dresner-Pollak R, Moses AC, Rosenblatt M, Zeind AJ, Clemens JD, Greenspan SL (1994) Specificity of urinary excretion of cross-linked N-telopeptides of type I collagen as a marker of bone turnover. Calcif Tissue Int 54(1):26-29

Yoshimura N, Muraki S, Oka H, Kawaquchi H, Nakamura K, Akune T (2011) Biochemical markers of bone turnover as predictors of osteoporosis and osteoporotic fractures in men and women: 10-year follow-up of the Taiji cohort. Mod Rheumatol 21(6):608-620, doi:10.1007/s10165-011-0455-2

doi:10.1186/2193-1801-3-577

Cite this article as: Addison et al:: Effects of de-escalated bisphosphonate therapy on bone turnover biomarkers in breast cancer patients with bone metastases. SpringerPlus 2014 3:577.

\section{Submit your manuscript to a SpringerOpen ${ }^{\circ}$ journal and benefit from:}

- Convenient online submission

- Rigorous peer review

- Immediate publication on acceptance

- Open access: articles freely available online

- High visibility within the field

- Retaining the copyright to your article

Submit your next manuscript at $\gg$ springeropen.com 